\title{
Novel methods of modulated-temperature thermal analysis ${ }^{1}$
}

\author{
Duncan M Price* \\ IPTME, Loughborough University, Loughborough Leicestershire LE11 3TU, UK
}

\begin{abstract}
Two novel methods of Modulated-Temperature Thermal Analysis are described. These are loosely-termed "Constrained Rate DSC" and "Modulated-Temperature TMA". In the first, the sample response is used to control the temperature program of a DSC in a method analogous to Controlled Rate Thermal Analysis. This results in a temperature modulation which is variable in period, amplitude and underlying heating rate depending on the sample behaviour and user defined limits. Deconvolution of the signals into thermally "reversing" and "non-reversing" heat flows is still possible. Secondly, the application of Modulated-Temperature Programming to Thermomechanical Analysis is discussed. (C) 1998 Elsevier Science B.V.
\end{abstract}

Keywords: Constrained rate calorimetry; Modulated-temperature TMA

\section{Introduction}

Modulated-temperature DSC employs a sinusoidal heating rate in place of a conventional linear temperature program in order to gain additional information about the thermal properties of materials. The signal from the instrument is separated into two components - a thermally "reversing" heat flow which is a function of temperature and heating rate, and a "nonreversing" heat flow which is a function of temperature and time. The former parameter is most readily identified with the heat capacity of the sample whereas, the non-reversing heat flow includes contributions from irreversible processes such as crystallization, chemical reactions and loss of volatile components.

Over a limited temperature range and assuming a linear instrumental response, the total heat flow

\footnotetext{
*Corresponding author. Fax: +44-1509-223949; e-mail: d.m.price@lboro.ac.uk

${ }^{1}$ Presented at TAC 97, Oxford, UK, 14-15 April 1997.
}

$(\mathrm{d} Q / \mathrm{d} t)$ from a DSC may be expressed as [1]:

$$
\mathrm{d} Q / \mathrm{d} t(T)=A(T) \times \mathrm{d} T / \mathrm{d} t+B(T, t)
$$

where $A(T)$ is the reversing heat flow and $B(T, t)$ is the non-reversing heat flow.

The modulated-temperature DSC experiment exposes the sample to a range of heating rates over a narrow temperature window and extracts the corresponding heating rate dependence of the heat flow to give the reversing component $A(T)$. The non-reversing component $B(T, t)$, is then found by the difference between the total heat flow and the product $A(T) \times \mathrm{d} T / \mathrm{d} t$. The concept of reversing and non-reversing thermal effects may be generalised to any thermal analysis technique where the measured property $(x)$ is considered to be made up of two contributions:

$$
\mathrm{d} x / \mathrm{d} t(T)=A^{\prime}(T) \times \mathrm{d} T / \mathrm{d} t+B^{\prime}(T, t)
$$

where $A^{\prime}(T)$ and $B^{\prime}(T, t)$ are the reversing and nonreversing components.

In principle such information could be obtained from several separate measurements on the same 
substance at different heating rates. This approach has most commonly been used to study the kinetics of thermal decomposition processes by thermogravimetry [2]. There are, however, obvious difficulties in obtaining a reproducible sample environment. Varying the heating rate in a continuous, periodic manner is a means of avoiding this problem and the approach behind modulated-temperature DSC.

In addition to sinusoidal heating rates, square or triangular waveforms have been employed [3]. These correspond to heat-isotherm and heat-cool temperature programs. Claudy et al. reported the use of the heat-isotherm mode (stepwise heating on cooling and warming) to study the glass transition of glycerol [4]. Their analysis divided the sample response into heat capacity (reversing) and time-dependent (non-reversing) contributions. Interestingly, this work did not use constant period temperature steps but limited the duration of the isothermal stage when the change in heat flow had fallen below a specified threshold. A similar approach has been reported by other workers for precise determination of transition temperatures for purity analysis and temperature calibration [5-8]. In 1967, Bean and Oliver disclosed a method for improving the resolution of differential thermal analysis measurements by constraining the temperature difference between sample and reference within predefined limits [9]. Thus, the heating rate was controlled by the sample response - a form of controlled rate thermal analysis [10]. Other workers have applied similar techniques to thermomechanical analysis and thermogravimetry $[11,12]$.

In this work the use of a DSC temperature program controlled by the sample response will be described and the application of modulated-temperature programming to thermomechanical analysis will be explored. For convenience, the two methods will be described as "Constrained Rate DSC" and "Modulated-Temperature TMA".

\section{Constrained rate DSC}

Measurements were carried out on a TA Instrument 2910 MDSC operated in conventional DSC mode. The method chosen exploited the "Abort next segment on limit" command to alternately heat the sample until the heat flow signal exceeded a pre-set limit and then
Table 1

Program listing for Constrained Rate DSC

\begin{tabular}{ll}
\hline 1. & Abort next segment if $\mathrm{W} / \mathrm{g}<$ limit \\
2. & Ramp rate ${ }^{\circ} \mathrm{C} / \mathrm{min}$ to final temperature ${ }^{\circ} \mathrm{C}$ \\
3. & Abort next segment if $\mathrm{W} / \mathrm{g}>$ limit \\
4. & Isothermal for $60 \mathrm{~min}$ \\
5. & Repeat segment 1 to final temperature ${ }^{\circ} \mathrm{C}$ \\
\hline
\end{tabular}

hold the temperature of the cell constant until the signal fell below this condition. The heat/isotherm segments were repeated until a suitable final temperature had been reached. A listing of the program is shown in Table 1, the key parameters which control the temperature profiles are the rate of the heating segment and the signal limit selected for the heat flow.

A plot of heat flow and heating rate vs. time for a sample of quench cooled poly(ethylene terephthalate) (PET) is shown in Fig. 1 for a rate of $10^{\circ} \mathrm{C} / \mathrm{min}$ and signal limit of $-0.2 \mathrm{~W} / \mathrm{g}$. Due to the thermal inertia of the system, the heat flow signal is not fixed at $-0.2 \mathrm{~W} /$ $\mathrm{g}$ but oscillates in a manner determined by the cell and sample. For the majority of the time the instrument is not operating under "true" DSC conditions but in a transient mode in which it is trying to achieve the programmed instructions, but either overshoots, or does not attain, a set condition. This results in an oscillating heating rate profile whose period does not change by a large amount, but whose amplitude and underlying heating rate are governed by the settings of rate and limit. This is illustrated for samples of PET in the temperature $150-200^{\circ} \mathrm{C}$ range (Table 2).

Deconvolution of the raw heat flow signal (time, temperature and heat flow) was carried out according to the method disclosed by Reading [13]. The heating rate was computed from the first derivative of the temperature-time data. The algorithm applied a linear least-squares fit to the heat flow vs. heating rate data over one cycle (i.e. successive minima or maxima in the heating rate) in accordance with Eq. (1). An adjustable parameter, the phase lag, is introduced to accommodate the delay between the heating rate and thermal response of the system. This was determined by shifting the heat flow data to one point at a time with respect to the heating rate until the best fit is achieved. Since this is an "offline" deconvolution method, the highest resolution is achieved by collect- 


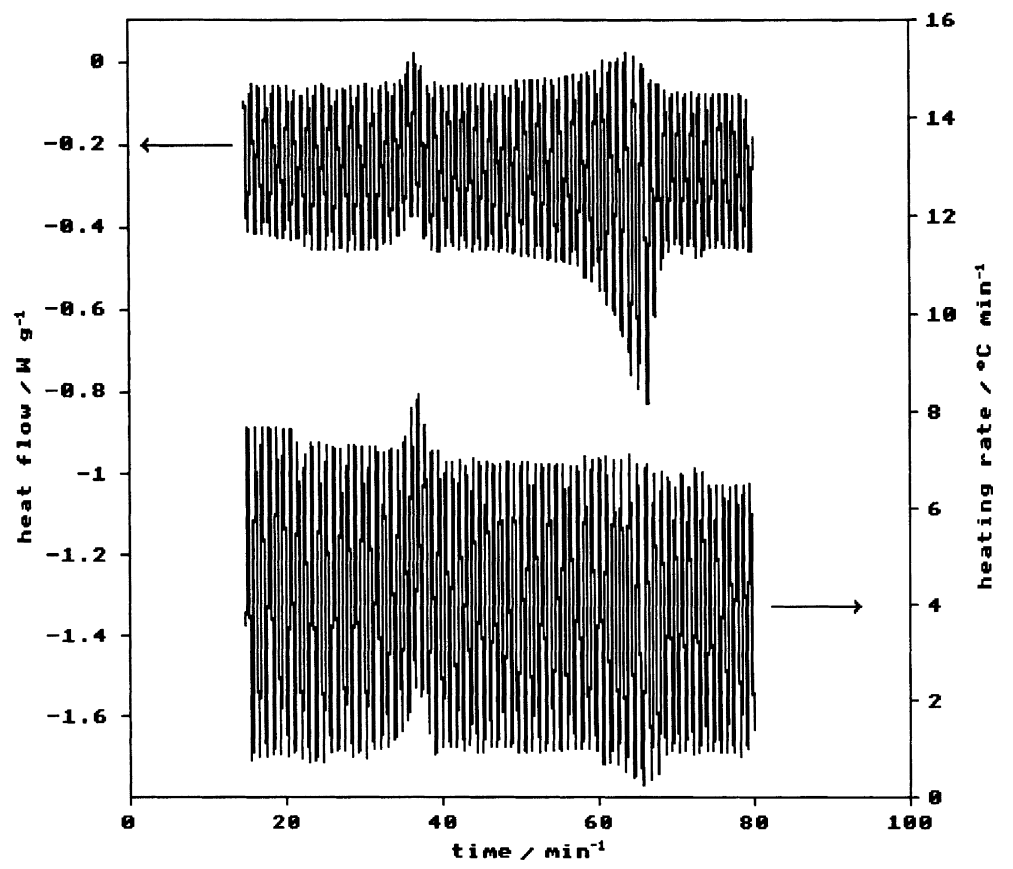

Fig. 1. Heat flow and heating rate for a sample of amorphous PET tested according to the temperature program in Table $1 .\left(\right.$ Rate: $10^{\circ} \mathrm{C} / \mathrm{min}$, limit: $-0.2 \mathrm{~W} / \mathrm{g}$.) Exothermic changes plotted in a upwards direction for Figs. 1-3.

Table 2

Effect of control parameters from Table 1 on temperature program

\begin{tabular}{lllll}
\hline Rate $\left({ }^{\circ} \mathrm{C} / \mathrm{min}\right)$ & Limit $(\mathrm{W} / \mathrm{g})$ & Period $(\mathrm{s})$ & Underlying heating rate $\left({ }^{\circ} \mathrm{C} / \mathrm{min}\right)$ & Amplitude $\left({ }^{\circ} \mathrm{C} / \mathrm{min}\right)$ \\
\hline 5 & 0.1 & 53 & 1.6 & 0.4 \\
10 & 0.1 & 56 & 3.3 & 0.7 \\
10 & 0.2 & 52 & 4.0 & 0.8 \\
\hline
\end{tabular}

ing data at short time intervals $(0.2 \mathrm{~s})$ although this increases the size of data files and analysis time.

Fig. 2 shows the underlying heat flow and heating rate profiles generated from the data in Fig. 1. Instead of being constant, the heating rate tracks the behaviour of the sample through the glass-rubber transition, cold crystallization and melting. The deconvoluted reversing and non-reversing heat flows are shown in Fig. 3. The results correspond with those obtained by conventional modulated-temperature studies, although there is perhaps a sharpening of the step change in reversing heat flow at the glass transition. Finally, the period of the temperature modulation and phase lag between the heating rate and heat flow response are shown in Fig. 4. Only in the melting region there is a significant change in these parameters.

\section{Modulated-temperature TMA}

Measurements were carried out on a Shimadzu TMA-50. A sample of $3 \mathrm{~mm}$ thick clear, unplasticised poly(vinylchloride) was placed beneath a $3 \mathrm{~mm}$ diameter flat-ended silica probe under a $200 \mathrm{~g}$ load. Measurements were started at $30^{\circ} \mathrm{C}$ followed by a succession of heat/hold/cool/hold stages which served to alternately raise the oven temperature by $5^{\circ} \mathrm{C}$ at $10^{\circ} \mathrm{C} / \mathrm{min}$ followed by $1 \mathrm{~min}$ isotherm, and then reduce the oven temperature by $2.5^{\circ} \mathrm{C}$ at the same rate, again followed by a $1 \mathrm{~min}$ isotherm. This cycle was repeated until a temperature of $150^{\circ} \mathrm{C}$ was reached. Sample length change and temperature were recorded by every second. The raw data from the instrument are shown in Fig. 5. 


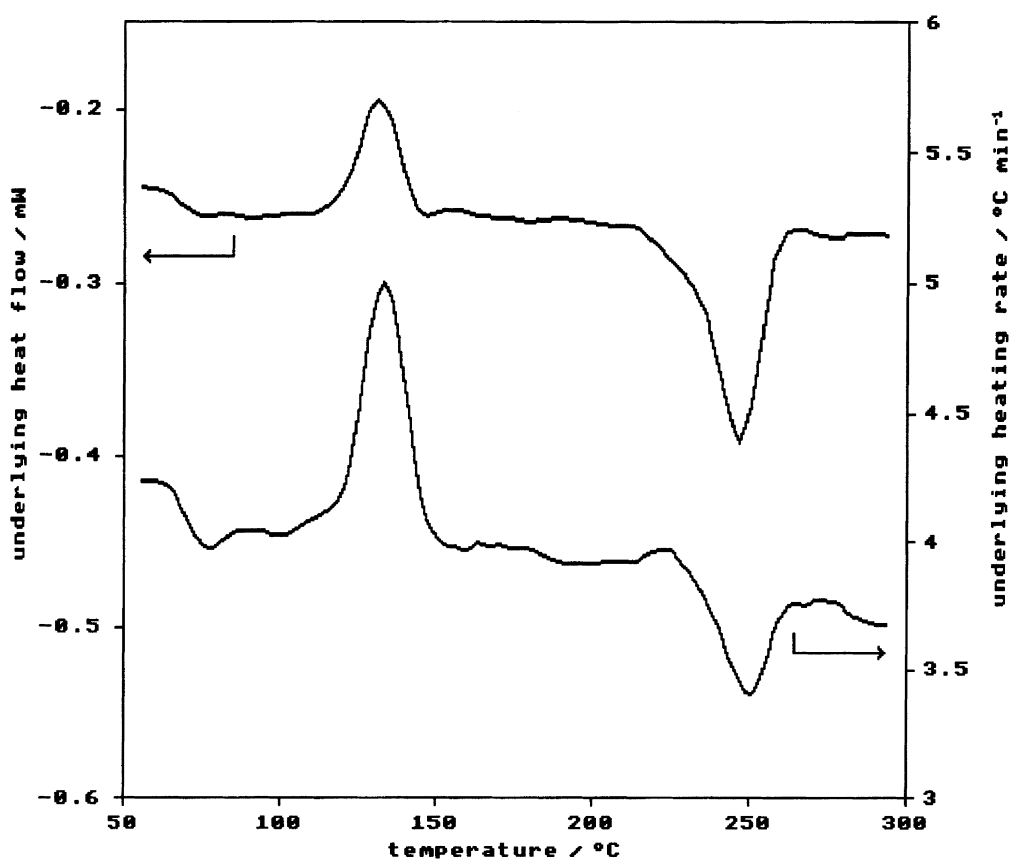

Fig. 2. Underlying heat flow and heating rate from the data in Fig. 1.

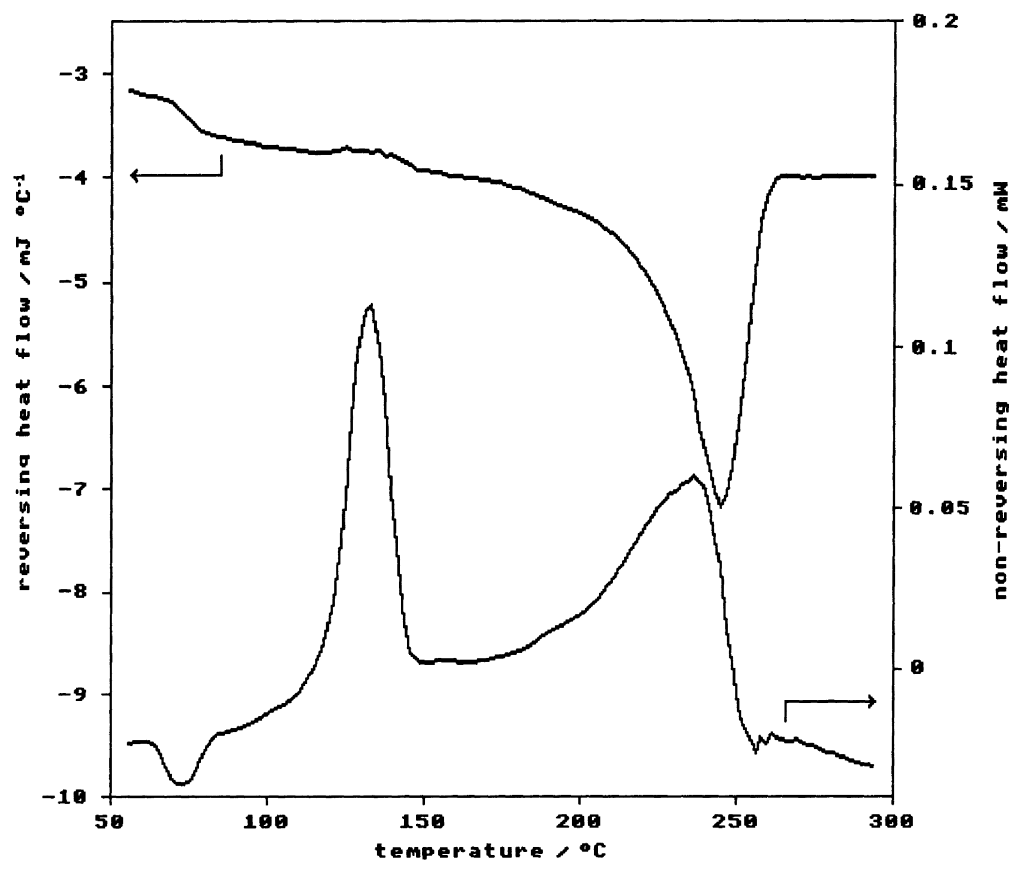

Fig. 3. Thermally reversing and non-reversing components of the data in Fig. 1. 


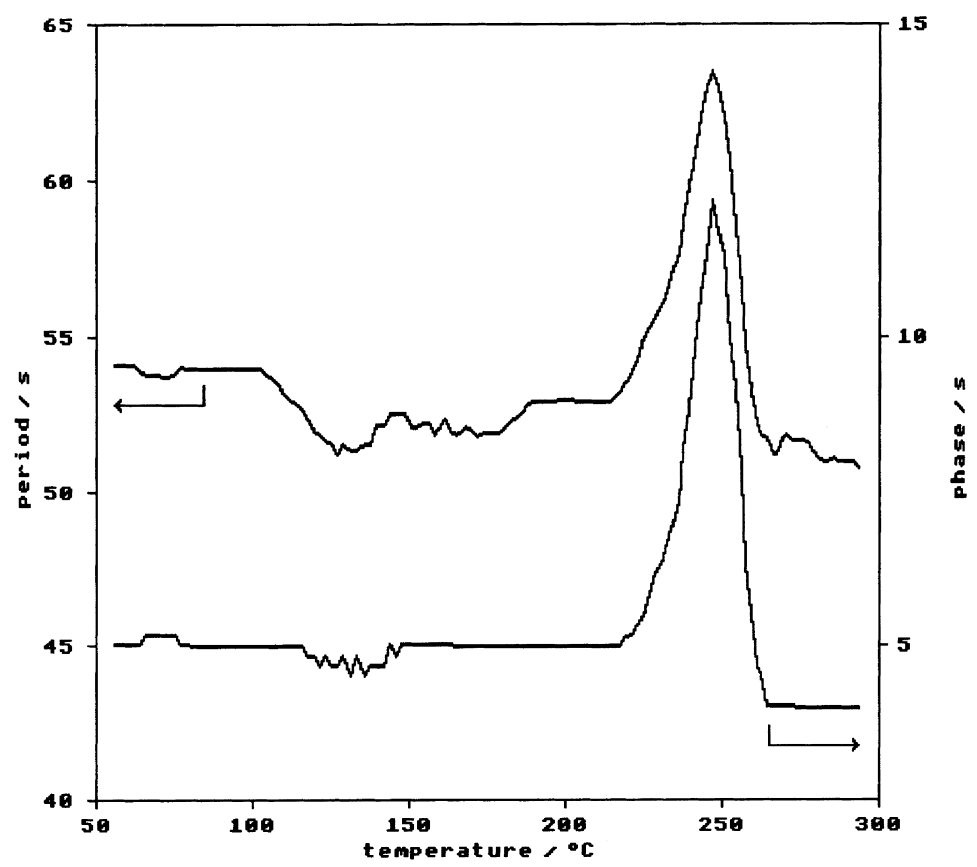

Fig. 4. Period of the temperature modulation and phase lag between heating rate and heat flow for the data in Fig. 1.

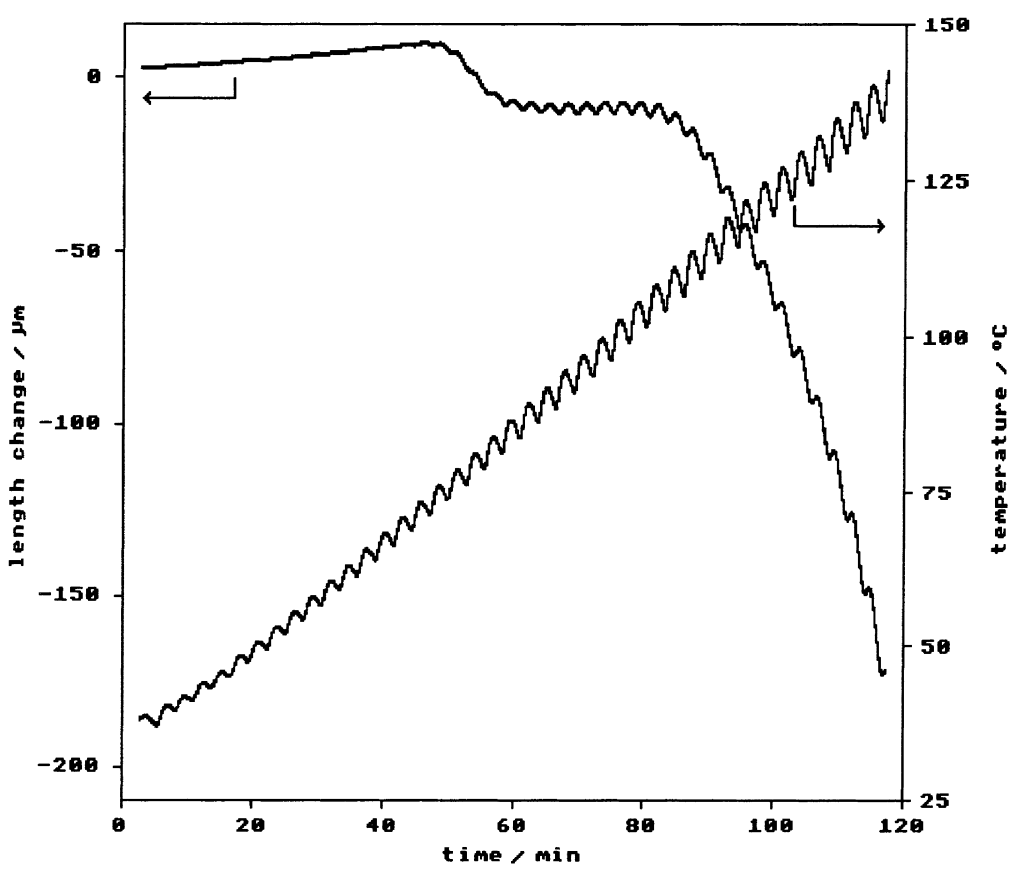

Fig. 5. Raw length change and temperature data for PVC (see text). Increase in sample length shown as a positive change. 


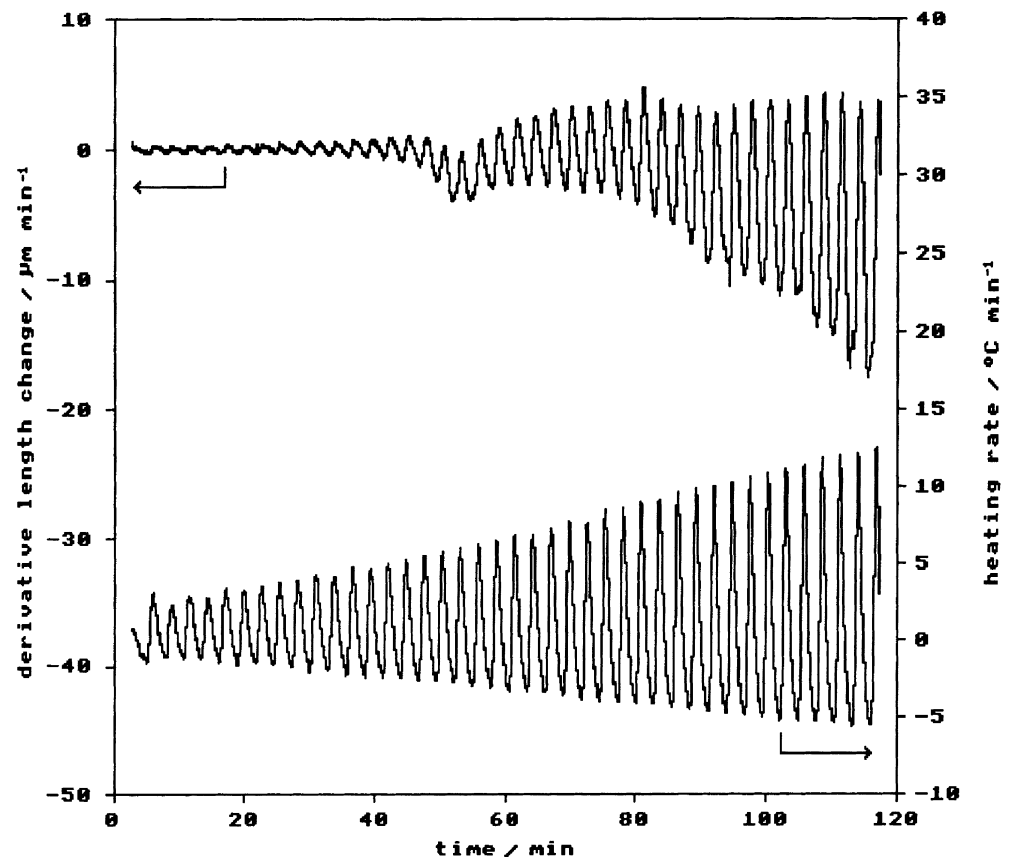

Fig. 6. First derivative of the length change and temperature profiles for the data in Fig. 5.

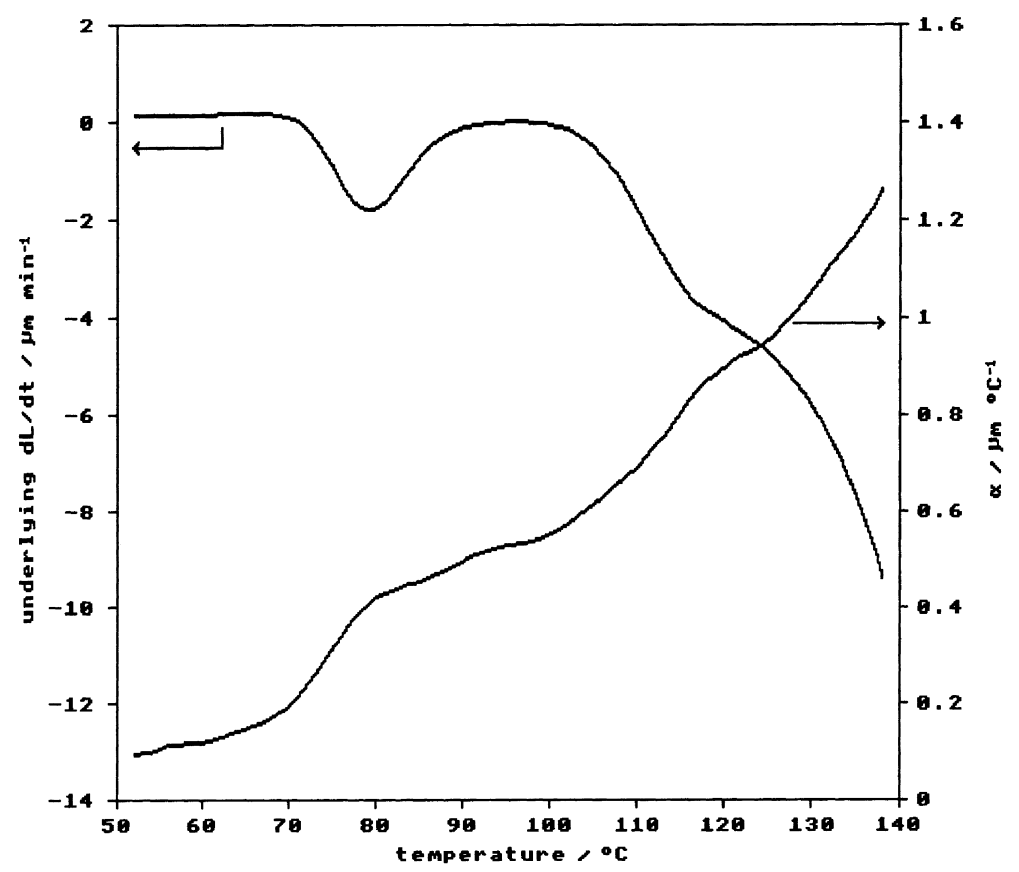

Fig. 7. Underlying derivative length change $(\mathrm{d} L / \mathrm{d} t)$ and thermal expansion coefficient $(\alpha)$ for PVC. 
The first derivatives of the length change and temperature $(\mathrm{d} T / \mathrm{d} t)$ curves are shown in Fig. 6 . The heating rate oscillates about a mean value of $0.91^{\circ} \mathrm{C} / \mathrm{min}$ with a $165 \mathrm{~s}$ period and, although heating rate amplitude increased during the experiment due to poor optimization of the temperature controller, the data could be deconvoluted by the same method used to analyse the DSC curve above, substituting the rate of change of sample length $(\mathrm{d} L / \mathrm{d} t)$ for the heat flow. In this case, the phase lag was found to be almost invariant throughout the experiment and a simpler deconvolution procedure could be employed whereby the underlying rate of change of sample length was found by averaging the data over one cycle and thermal expansion coefficient $(\alpha)$ was determined from the ratio of the amplitude of the oscillating component of the $\mathrm{d} L / \mathrm{d} t$ signal divided by the amplitude of the oscillating heating rate (Fig. 7).

These quantities can be integrated with respect to time (for the underlying $\mathrm{d} L / \mathrm{d} t$ ) or temperature (for $\alpha$ ) to allow the total length change and length change due to thermal expansion to be determined (Fig. 8). The difference between the two curves represents the amount of penetration of the probe into the sample due to softening and contains information pertaining to the viscoelastic properties of the material. Since the heating program exposes the sample to a succession of heat/cool/re-heat cycles, it is possible to separately analyse each part of the temperature profile in order to study the effect of thermal history of the sample response (Reading has termed this "parsing" [13]). Treatment of the data in this manner indicates that the bulk of the sample's softening under load occurs during the first heating part of the temperature cycle and that thermal expansion is largely unaffected by the direction of the temperature change except in the glass-transition region where relaxation effects are evident.

\section{Conclusions}

These studies indicate that it is not necessary to employ a constant period, amplitude and underlying heating rate for modulated-temperature DSC. Instead, the sample response can be used to dictate the course of the temperature profile. This has potential benefits for improving the resolution of the technique and may find application for calibration purposes or purity

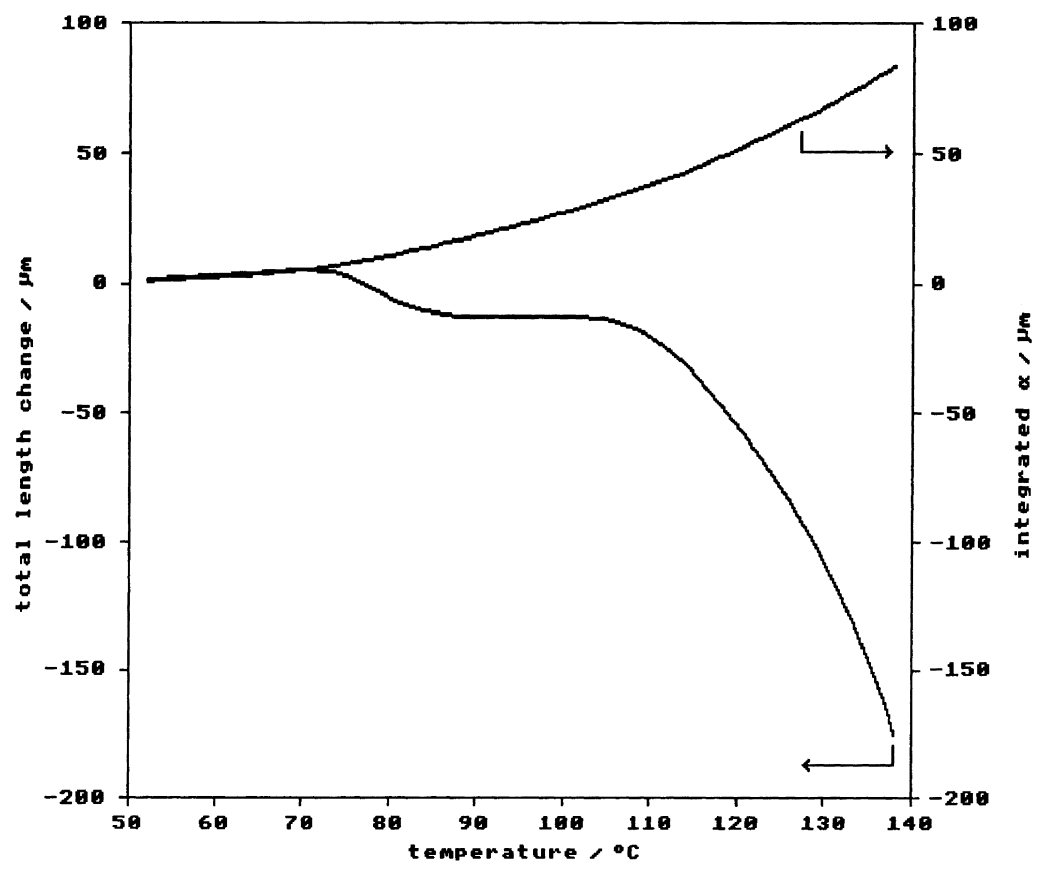

Fig. 8. Cumulative integrals of the data in Fig. 7 showing the total length change and change in length due to thermal expansion (integrated $\alpha$ ) of PVC. The difference between both curves represents the depth of penetration into the sample under the applied load. 
analysis. Modulated-temperature programs may also be used in thermomechanical analysis to separate the reversible changes that accompany thermal expansion from the irreversible effects that occur when a sample softens under load. The same principles might also be employed in dynamic mechanical analysis and in thermogravimetry.

\section{Acknowledgements}

The TMA measurements were carried out at the Thermal Methods and Conservation Science Laboratory, Department of Chemistry, Birkbeck College, University of London. The author is grateful to Marianne Odlyha and Gary Foster for their hospitality and to Shimadzu (UK) Ltd. for the loan of equipment.

\section{References}

[1] M. Reading, Trends Polym. Sci. 1 (1993) 248.

[2] T. Ozawa, Bull. Chem. Soc. Jpn. 38 (1965) 1881.
[3] R. Reisen, G. Widmann, R. Truttmann, Thermochim. Acta 272 (1996) 27.

[4] P. Claudy, J.C. Commerceron, J.M. Létoffé, Thermochim. Acta 128 (1988) 251.

[5] H. Staub, W. Perron, Anal. Chem. 46 (1974) 128.

[6] J.H. Flynn, Thermochim. Acta 8 (1974) 69.

[7] K.A. Simonsen, M. Zaherescu, J. Thermal Anal. 15 (1979) 25.

[8] G. Kiss, K. Seybold, T. Meisel, in: H.G. Wiedemann (Ed.), Thermal analysis, Proc. 6th Int. Conf. Thermal Anal., Bayreuth, FRG, Birkhäuser Verlag, Basel, 1980, p. 8792.

[9] C.M. Bean, R.H. Oliver, British Patent 1,063,898, 1967.

[10] M. Reading, in: E.L. Charsley, S.B. Warrington (Eds.), Thermal Analysis - Techniques and Applications, The Royal Society of Chemistry, Cambridge, 1992, pp. 127155 .

[11] O. Toft Sørensen, Thermochim. Acta 50 (1981) 163.

[12] F. Paulik, Special Trends in Thermal Analysis, John Wiley and Sons, Chichester, 1995.

[13] M. Reading, US Patent 5,474,385, 1995. 